\title{
A Large Radio Detector at the Pierre Auger Observatory - Measuring the Properties of Cosmic Rays up to the Highest Energies
}

Bjarni Pont $^{* a}$ for the Pierre Auger Collaboration ${ }^{\dagger b}$

${ }^{a}$ Department of Astrophysics/IMAPP, Radboud University, P.O. Box 9010, NL-6500 GL

Nijmegen, The Netherlands

${ }^{b}$ Observatorio Pierre Auger, Av. San Martín Norte 304, 5613 Malargüe, Argentina

E-mail: auger_spokespersons@fnal.gov

Full author list: http://www.auger.org/archive/authors_icrc_2019.html

High-energy cosmic rays impinging on the atmosphere of the Earth induce cascades of secondary particles, the extensive air showers. Many particles in the showers are electrons and positrons, which due to interactions with the magnetic field of the Earth emit radiation with frequencies of several tens of MHz. In the last years, huge progress has been made in measuring the characteristics of extensive air showers through their radio signal at these frequencies.

The radio technique is now routinely applied to measure the properties of cosmic rays, such as their arrival direction, their energy, and their particle type/mass. Air showers with zenith angles above $60^{\circ}$ have a large footprint of the radio emission on the ground which can be detected with sparse arrays with kilometer-scale spacing. With the Auger Engineering Radio Array (AERA) these "horizontal air showers" are measured, demonstrating the feasibility of the radio technique for highly inclined showers.

At present, the Auger Collaboration is upgrading its detectors. The upgrade includes the installation of radio antennas on each of the 1661 water-Cherenkov detectors of the array. The main objective of the radio upgrade (Radio Detector) is to measure horizontal air showers and to determine the properties of cosmic rays up to the highest energies. The combination of waterCherenkov detectors and radio antennas will provide muon-electron separation for horizontal air showers at the highest energies.

Details of the technical implementation and the expected performance will be presented.

36th International Cosmic Ray Conference - ICRC2019

24 July - 1 August, 2019

Madison, Wisconsin, USA

\footnotetext{
${ }^{*}$ Speaker.

${ }^{\dagger}$ for collaboration list see PoS(ICRC2019)1177
} 


\section{Introduction}

The Pierre Auger Observatory, located in western Argentina, is the world's largest cosmicray Observatory [1]. The objectives of the Observatory are to probe the origin and characteristics of cosmic rays above $10^{17} \mathrm{eV}$ and to study the interactions of these, the most energetic particles observed in Nature. The Auger design features an array of 1600 water-Cherenkov detector stations on a $1500 \mathrm{~m}$ grid, spread over $3000 \mathrm{~km}^{2}$, and overlooked by 24 air fluorescence telescopes. In addition, three high-elevation fluorescence telescopes overlook a $23.5 \mathrm{~km}^{2}, 61$-detector infilled array with $750 \mathrm{~m}$ spacing. Radio emission from extensive air showers is measured with the Auger Engineering Radio Array (AERA), comprising more than 150 radio detector stations, covering an area of about $17 \mathrm{~km}^{2}$, co-located with the infill array [2, 3].

At present, the Pierre Auger Collaboration is working on an upgrade of the Observatory, Auger Prime [4]. The physics case of the upgrade is outlined in [5]. The key science questions to be addressed are: What are the sources and acceleration mechanisms of ultra-high-energy cosmic rays (UHECRs)? Do we understand particle acceleration and physics at energies well beyond the LHC (Large Hadron Collider) scale? What is the fraction of protons, photons, and neutrinos in cosmic rays at the highest energies?

To achieve these objectives, a layer of scintillators is being installed above the water-Cherenkov detectors (the Surface Scintillator Detector), the observation time of the fluorescence detectors is being increased, and underground muon detectors are being installed in a part of the Surface Detector array. In addition, radio antennas will be added to each Surface Detector station - this is the focus of this contribution.

We are going to install a radio antenna on each of the 1661 stations of the Surface Detector array of the Observatory, forming a $3000 \mathrm{~km}^{2}$ radio array, the largest radio array for cosmic-ray detection in the world. A photograph of a prototype station is shown in Fig. 1. It shows (from bottom to top) the water-Cherenkov detector with a layer of scintillators on top (Surface Scintillator Detector) and a radio antenna (Radio Detector). The concept of radio antennas on top of the Surface Detector stations has been studied earlier at the Auger Observatory on smaller scales [6, 7]. With the combination of water-Cherenkov detector and Surface Scintillator Detector the electron-tomuon ratio $(\mathrm{e} / \mu)$ is measured for vertical showers. In a similar way the combination of waterCherenkov detector and Radio Detector will be used to measure the ratio of the electromagnetic energy and the number of muons for horizontal air showers. Thus, the Radio Detector will increase the aperture of the Observatory for mass-sensitive investigations, allowing the $\mathrm{e} / \mu$ separation for showers with a broad zenith angle range, from zenith with the Surface Scintillator Detector to the horizon with the Radio Detector.

Horizontal air showers have a large footprint of the radio emission on the ground, covering areas of the order of $100 \mathrm{~km}^{2}$ for very inclined showers. This has been predicted by simulations [ 8 , 9] and also recently been measured with AERA [10,11, 12]. Thus, the radio emission from such showers can be recorded with a sparse antenna array with $1.5 \mathrm{~km}$ spacing. 

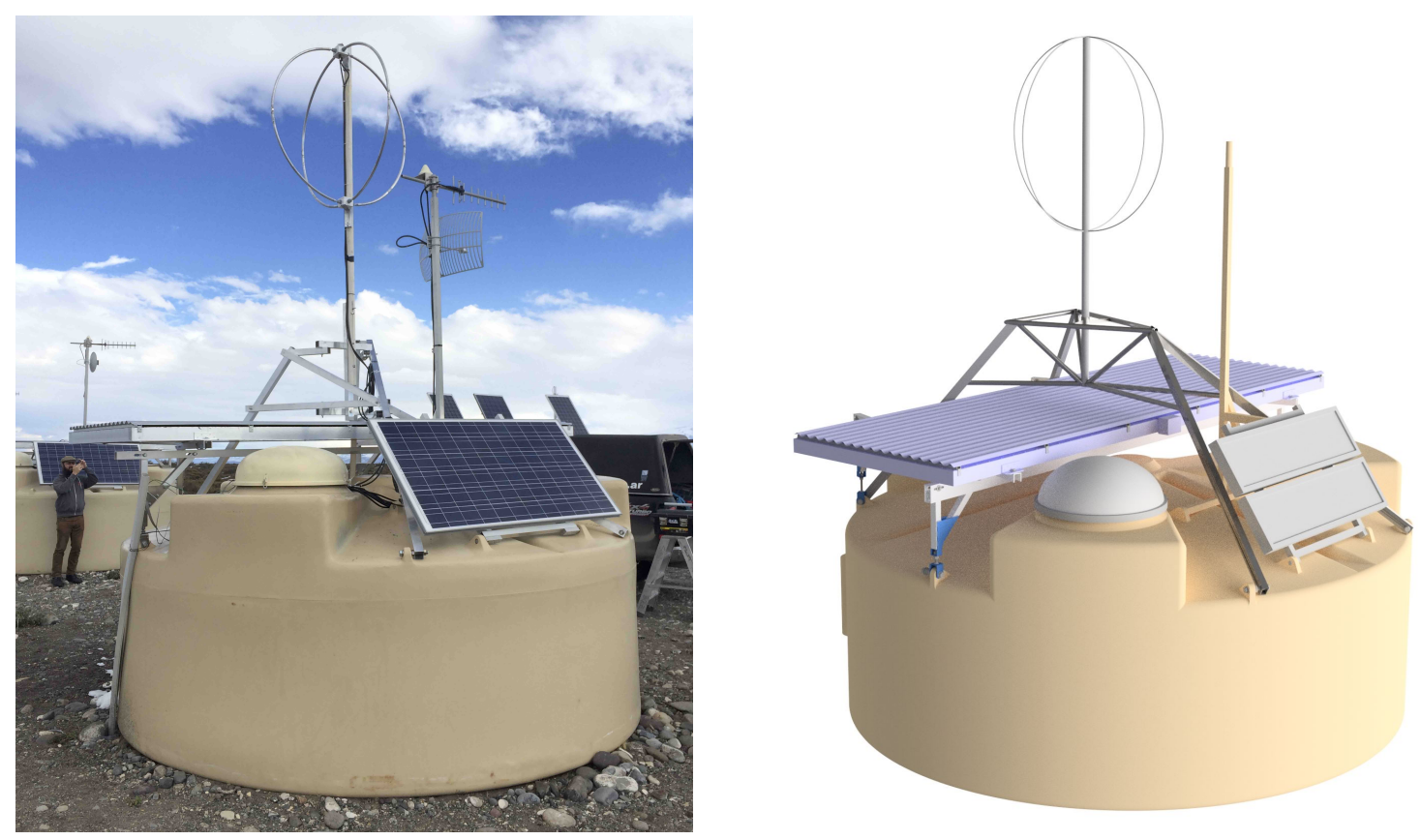

Figure 1: An upgraded station of the Surface Detector array of the Pierre Auger Observatory. On top of the water-Cherenkov detector a layer of scintillators (Surface Scintillator Detector - SSD) and a radio antenna (Radio Detector - RD) are mounted. Left: photograph of a prototype station, right: schematic view.

\section{The Radio Detector of the Pierre Auger Observatory}

At present we are working on the details of the technical implementation of the Radio Detector. The photograph (Fig. 1) depicts a prototype, which is installed at the observatory since May 2019. The Radio Detector will be fully integrated in the Surface Detector stations, they will form one unit, being comprised of water-Cherenkov detector, Surface Scintillator Detector, and Radio Detector. The different detectors will share the infrastructure such as solar power, battery, communications system, GPS timing, and an integrated data acquisition system. The envisaged system is schematically shown in Fig. 2.

We aim to use a short aperiodic loaded loop antenna (SALLA) to detect the radio emission from air showers in the frequency range 30 to $80 \mathrm{MHz}$. The SALLA realizes a Beverage antenna as a dipole loop of $1.2 \mathrm{~m}$ diameter $[13,14]$. The SALLA has been developed to provide a minimal design that fulfills the need for both, ultra-wideband sensitivity, and low costs for production and maintenance of the antenna in a large-scale radio detector. The compact structure of the SALLA makes the antenna robust and easy to manufacture. Beverage antennas include a resistor load within the antenna structure to give a specific shape to the directivity [14]. In the case of the SALLA a resistance of $500 \Omega$ connects the ends of the dipole arms at the bottom of the antenna. The antenna is read out at the top which is also the position of the Low-Noise Amplifier (LNA). While signals coming from above will induce a current directly at the input of the amplifier, the reception from directions below the antenna is strongly suppressed as the captured power is primarily consumed within the ohmic resistor rather than amplified by the LNA. The resulting strong 


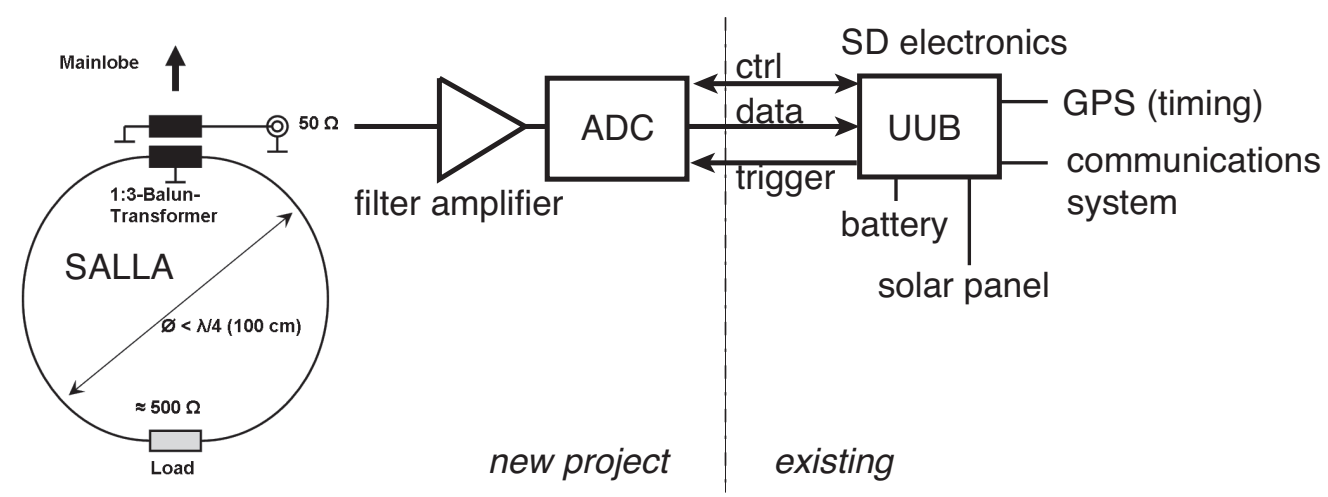

Figure 2: Schematic view of the envisaged read out for the radio antennas. The radio antenna (SALLA) is read out through a filter amplifier and an ADC. The front-end board has an interface to the existing electronics (Upgraded Unified Board - UUB [16]) at each Surface Detector station.

suppression of sensitivity towards the ground reduces the dependence of the antenna on structures below the antenna (like the Surface Scintillator Detector and the water-Cherenkov detector) and on environmental conditions which might vary as a function of time and are thus a source of systematic uncertainty. With the inclusion of an ohmic resistor the SALLA especially challenges its amplifier as only $\sim 10 \%$ of the captured signal intensity is available at the input of the LNA. Proper matching between the antenna structure and the LNA is realized with a 3:1 transmission line transformer. The structure of the SALLA creates a sensitivity which is flat as a function of frequency.

The water-Cherenkov detector will issue a trigger signal when energy deposition has been detected. The data from the radio antenna will be passed to the read-out electronics of the Surface Scintillator Detector/water-Cherenkov detector system (UUB) [16] and will be transmitted together with all data from the station to the central data acquisition of the Auger Observatory.

We foresee two polarization directions of the antenna, oriented orthogonal to each other (see also Fig. 1). The signals of the two analogue channels will be pre-amplified in a LNA at the antenna. The signals are transmitted through shielded coaxial cables to the filter amplifier on the front-end board. They will be digitized with a sampling frequency of 200 Msps. A FPGA controls the data flow and the communication with the existing electronics of each Surface Detector station (UUB).

\section{Expected performance}

We first illustrate the estimated aperture and event statistics achievable with the Radio Detector. These were derived with a Monte Carlo study on the basis of an analytical signal model [15] based on CoREAS simulations [17]. We have expanded the signal model to enable predictions for zenith angles beyond $60^{\circ}$. This expansion entails the continuation of the spline fits relating the parameters $R, \sigma$ and $k$ to the distance to shower maximum $D_{X_{\max }}$ (cf. Fig. 8 of reference [15]) using CoREAS simulations with zenith angles up to $80^{\circ}$. On the basis of predicted signals for varying energies and event geometries, we determine detection efficiencies, requiring an energy fluence of at least $5 \mathrm{eV} / \mathrm{m}^{2}$ in at least three antennas on a $1.5 \mathrm{~km}$ triangular grid. The estimate of $5 \mathrm{eV} / \mathrm{m}^{2}$ for the detection threshold was obtained with SALLA prototype stations deployed within AERA 

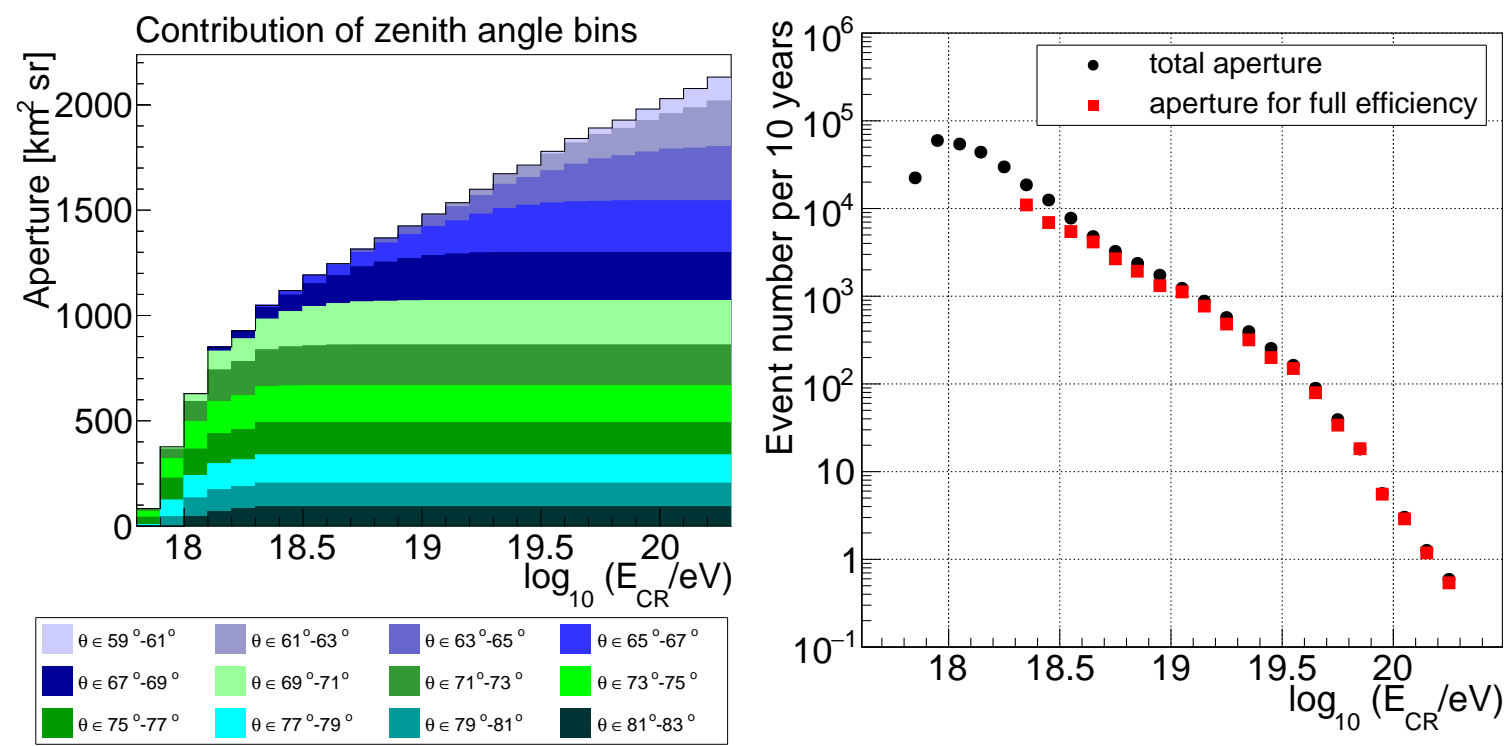

Figure 3: Left: Predicted aperture of the Radio Detector as a function of cosmic-ray energy for different ranges of zenith angles. Detection efficiencies were determined with the requirement of an energy fluence (i.e., energy deposit in the form of radio waves per unit ground area) of at least $5 \mathrm{eV} / \mathrm{m}^{2}$ in at least three antennas, and with shower cores contained in the geometrical area of the $3000 \mathrm{~km}^{2}$ array.. Right: Number of events measured with the Radio Detector as a function of energy over the course of 10 years, calculated from the aperture using the Auger energy spectrum. Black points correspond to the total aperture as shown in the left panel. Red points correspond to the aperture in regions of parameter space where detection is $100 \%$ efficient (for every energy bin only those zenith angles are counted where full efficiency has already been reached).

in 2017. For comparison, the LPDA and butterfly antennas in AERA have a threshold of approximately $2 \mathrm{eV} / \mathrm{m}^{2}$. Assuming instrumentation of $3000 \mathrm{~km}^{2}$ and requiring shower cores to fall within this geometrical area ${ }^{1}$, we derive the aperture as displayed in Fig. 3.

It is visible that the energy threshold for coincident detection of showers in at least three antennas decreases with increasing zenith angle. This is due to the growth of the radio-emission footprint with zenith angle. The aperture contribution at the highest zenith angles is, however, limited by the condition that the events need to be contained in the geometric area of the Observatory, which introduces a $\cos \theta$ factor. At lower zenith angles, in particular below $70^{\circ}$ zenith angle, the maximum achievable aperture contribution is larger, yet the detection becomes fully efficient only at high energies. A lower detection threshold than the assumed $5 \mathrm{eV} / \mathrm{m}^{2}$, possibly achievable using more sophisticated signal cleaning techniques, would thus in particular increase the fully efficient aperture contributed by air showers with low zenith angles. We note that the aperture shown in figure 3 should be considered a "detection aperture" and not a "trigger aperture", as the trigger will be provided by the Surface Detector. Radio stations with a signal below $5 \mathrm{eV} / \mathrm{m}^{2}$ will thus also contribute useful information.

Multiplying the aperture with the flux as measured by the Pierre Auger Observatory [19], we

\footnotetext{
${ }^{1}$ We emphasize that this is a conservative scenario, as also air showers without core in the geometrical area of the observatory could be detected; cf. the science case of GRAND [18].
} 


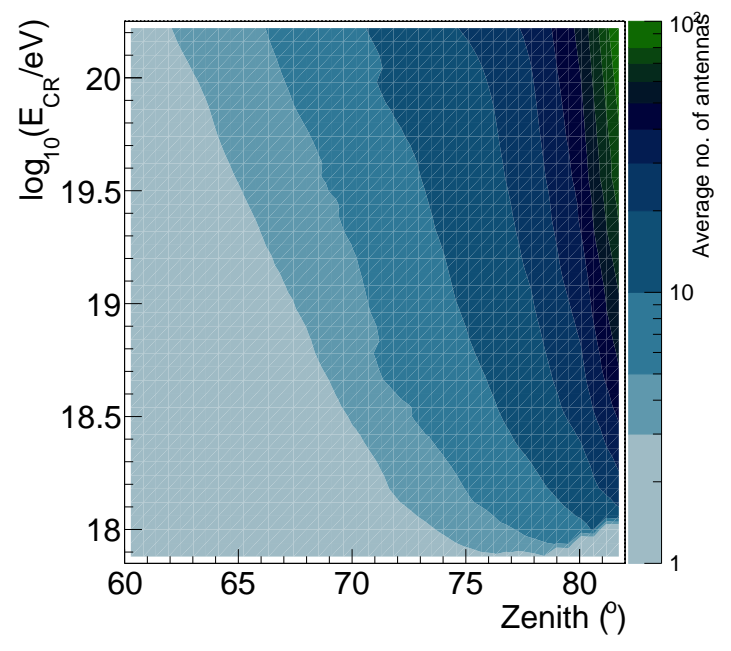

Figure 4: Average number of radio stations with a signal above the detection threshold of $5 \mathrm{eV} / \mathrm{m}^{2}$ as a function of zenith angle and cosmic-ray energy.

derive the expected number of cosmic rays for the envisaged lifetime of the Radio Detector of 10 years and display it in Fig. 3. Over this period, more than 3000 cosmic rays will be measured at energies exceeding $10^{19} \mathrm{eV}$, and approximately 300 air showers will exceed an energy of $10^{19.5} \mathrm{eV}$. We note that an increase of the assumed detection threshold mostly affects detection efficiencies and thus event rates at energies below $10^{19} \mathrm{eV}$. To convey an impression of how well the events will be sampled with the Radio Detector, we have also determined the average number of radio stations with a signal above $5 \mathrm{eV} / \mathrm{m}^{2}$ as a function of zenith angle and cosmic-ray energy, shown in figure 4 .

To illustrate the expected performance, we pick a particular physics case: The Radio Detector will be a powerful tool to study the muon content in inclined air showers. The almost pure separation of the muon content (water-Cherenkov detectors) and electromagnetic energy (radio antennas) of inclined air showers will allow us to determine the energy dependence of the number of muons in inclined air showers, as has previously been done with the combination of water-Cherenkov Detectors and Fluorescence Detectors (FD) [21, 20]. With the Radio Detector, however, we will achieve an order of magnitude higher event statistics: To date, a total of 202 hybrid FD-SD events above $10^{18.8} \mathrm{eV}$ have been collected for use in this analysis [20]. Over the lifetime of the Radio Detector, we estimate to collect roughly 6500 air showers in that energy range.

To illustrate the potential, we have performed a simulation study in which we draw events according to the distribution shown in Fig. 3 (red points, i.e., requiring full efficiency). We then smear out the energy, which will be measured by the Radio Detector, by a Gaussian with a $\sigma$ of $20 \%$, i.e., using a conservative scenario for the energy resolution. We draw muon numbers, taking into account the measured mean number of muons as a function of energy and their measured intrinsic spread above $10^{18.8} \mathrm{eV}$ as taken from reference [20]. Furthermore, we smear out the number of muons by the $N_{19}$ measurement resolution as detailed in [22]. Afterwards, we bin the data as a function of (smeared) energy and determine the mean number of muons, shown in Fig. 5 (left), as well as the intrinsic spread of the number of muons (after subtracting the $N_{19}$ measurement resolution), shown in Fig. 5 (right).

It becomes obvious that with the vastly increased statistics available through the Radio Detector, a powerful measurement of the mean number of muons as well as their intrinsic spread as a 

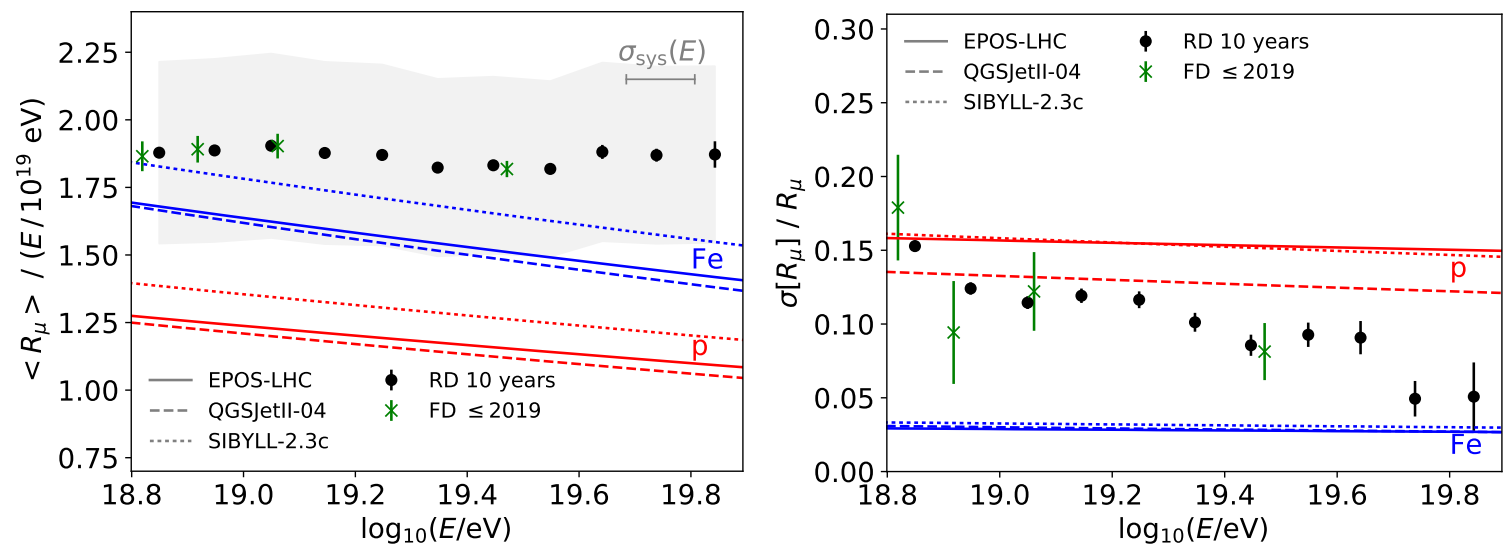

Figure 5: Simulation of a measurement of the mean number of muons (left) and the spread of the number of muons (right) as a function of energy via combined measurements with the water-Cherenkov Detector and the Radio Detector. A conservative energy resolution of $20 \%$ has been assumed for the radio measurement. Error bars indicate statistical uncertainties. Green points indicate the currently available data [20]. Black points indicate data as measurable with the Radio Detector over the course of 10 years. The shaded area indicates the systematic uncertainty of the measurement arising from the Auger energy scale and the systematic uncertainty of the muon measurement. Lines indicate model predictions for EPOS-LHC (solid), QGSJETII-04 (dashed), and Sibyll-2.3c (dotted).

function of cosmic-ray energy will be possible. The latter measurement, in particular, will allow in-depth tests of hadronic interactions.

We note that the systematic uncertainty illustrated in the form of a grey band in Fig. 5 (left) is dominated by the $14 \%$ uncertainty of the energy scale of the Pierre Auger Observatory. The Radio Detector has potential to validate and improve the energy scale determination up to the highest energies (similar to earlier investigations for vertical air showers [23, 24]), which would help further to perform more stringent comparisons of existing hadronic interaction models with data.

\section{Conclusion and outlook}

We are at present finalizing the technical design of the Radio Detector of the Auger observatory. First prototypes of all components are installed in the field for an in-situ evaluation of their performance. We aim to start the mass production of the components towards the end of 2019 and to complete the deployment of the almost 1700 units of the $3000 \mathrm{~km}^{2}$ Radio Detector before the end of 2021. The Radio Detector will improve the science capabilities of the Auger observatory, by providing electron-muon separation for inclined air showers up to the highest energies. This implies also a larger mass-sensitive set of cosmic rays, measured by the Auger observatory in the overlap region on the sky with the Telescope Array.

The Auger Radio Detector is a natural next step towards future cosmic-ray experiments, applying the radio technique on even larger scales, such as GRAND [18] or a next-generation cosmic ray experiment, applying hybrid detection techniques by combining radio antennas with e.g. segmented water-Cherenkov detectors [25]. The Radio Detector with a $3000 \mathrm{~km}^{2}$ array is a logical step 
between the presently largest array (AERA, $17 \mathrm{~km}^{2}$ ) and applications of the technique on a level of tens of thousands of $\mathrm{km}^{2}$. The Auger Radio Detector will be the biggest array for the next decade, allowing us to evaluate the detector technology, establish reconstruction methods, and study the physics performance of such large radio arrays.

\section{References}

[1] A. Aab [Pierre Auger Collaboration], Nucl. Instrum. Meth. A 798 (2015) 172-213.

[2] J. Schulz [Pierre Auger Collaboration], Proceedings of the 34th ICRC Den Haag (2015) POS (ICRC2015) 615.

[3] T. Huege [Pierre Auger Collaboration], EPJ Web Conf. 210 (2019) 05011.

[4] A. Castellina [Pierre Auger Collaboration], EPJ Web Conf. 210 (2019) 06002.

[5] A. Aab [Pierre Auger Collaboration], arXiv (2016) 1604.03637.

[6] C. Berat [Pierre Auger Collaboration], Nucl. Instr. Meth. A 718 (2013) 471.

[7] R. Gaior, PhD thesis, https://hal.archives-ouvertes.fr/tel-01826441.

[8] T. Huege, Phys. Rept. 620 (2016) 1-52.

[9] F. Schröder, Prog.Part.Nucl.Phys. 93 (2017) 1-68.

[10] A. Aab [Pierre Auger Collaboration], JCAP 1810 (10) (2018) 026.

[11] M. Gottowik [Pierre Auger Collaboration], Proceedings ARENA 2018 (2018).

[12] M. Gottowik [Pierre Auger Collaboration], Proceedings of the 36th ICRC, Madison, POS ( ICRC2019) 274, 2019.

[13] O. Krömer, et al., Proceedings of the 31st ICRC, Lodz, Poland, 1232, 2009.

[14] P. Abreu [Pierre Auger Collaboration], JINST 7 (2012) P10011.

[15] C. Glaser, S. de Jong, M. Erdmann, J. R. Hörandel, Astropart. Phys. 104 (2019) 64-77.

[16] D. Nitz [Pierre Auger Collaboration], Proceedings of the 36th ICRC, Madison, POS (ICRC2019) 370, 2019.

[17] T. Huege, M. Ludwig, C. W. James, AIP Conf. Proc. 1535 (2013) 128.

[18] J. Alvarez-Muñiz, et al., Science China: Phys., Mech. \& Astron., 63, 219501 (2020) http://arxiv.org/abs/1810.09994

[19] F. Fenu [Pierre Auger Collaboration], PoS (ICRC2017),486(2018).

[20] F. Riehn [Pierre Auger Collaboration], Proceedings of the 36th ICRC, Madison, POS (ICRC2019) 404, 2019.

[21] A. Aab [Pierre Auger Collaboration], Phys. Rev. D91 (3) (2015) 032003, [Erratum: Phys. Rev.D91,no.5,059901(2015)].

[22] A. Aab [Pierre Auger Collaboration], JCAP 1408 (08) (2014) 019.

[23] A. Aab [Pierre Auger Collaboration], Phys. Rev. D 93 (12) (2016) 122005.

[24] A. Aab [Pierre Auger Collaboration], Phys. Rev. Lett. 116 (24) (2016) 241101.

[25] J. Hörandel, Proceedings ARENA 2018 (2018). 